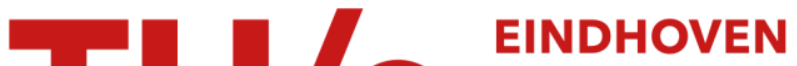 UNIVERSITY OF TECHNOLOGY
}

\section{Impact analysis of directional antennas and multiantenna beamformers on radio transmission}

Citation for published version (APA):

Yang, H., Herben, M. H. A. J., Akkermans, J. A. G., \& Smulders, P. F. M. (2008). Impact analysis of directional antennas and multiantenna beamformers on radio transmission. IEEE Transactions on Vehicular Technology, 57(3), 1695-1707. https://doi.org/10.1109/TVT.2007.907308

DOI:

10.1109/TVT.2007.907308

Document status and date:

Published: 01/01/2008

Document Version:

Publisher's PDF, also known as Version of Record (includes final page, issue and volume numbers)

Please check the document version of this publication:

- A submitted manuscript is the version of the article upon submission and before peer-review. There can be important differences between the submitted version and the official published version of record. People interested in the research are advised to contact the author for the final version of the publication, or visit the $\mathrm{DOI}$ to the publisher's website.

- The final author version and the galley proof are versions of the publication after peer review.

- The final published version features the final layout of the paper including the volume, issue and page numbers.

Link to publication

\section{General rights}

Copyright and moral rights for the publications made accessible in the public portal are retained by the authors and/or other copyright owners and it is a condition of accessing publications that users recognise and abide by the legal requirements associated with these rights.

- Users may download and print one copy of any publication from the public portal for the purpose of private study or research.

- You may not further distribute the material or use it for any profit-making activity or commercial gain

- You may freely distribute the URL identifying the publication in the public portal.

If the publication is distributed under the terms of Article 25fa of the Dutch Copyright Act, indicated by the "Taverne" license above, please follow below link for the End User Agreement:

www.tue.nl/taverne

Take down policy

If you believe that this document breaches copyright please contact us at:

openaccess@tue.nl

providing details and we will investigate your claim. 


\title{
Impact Analysis of Directional Antennas and Multiantenna Beamformers on Radio Transmission
}

\author{
Haibing Yang, Member, IEEE, Matti H. A. J. Herben, Senior Member, IEEE, \\ Iwan J. A. G. Akkermans, Student Member, IEEE, and Peter F. M. Smulders, Senior Member, IEEE
}

\begin{abstract}
The impact of directional antennas and multiantenna beamformers on radio transmission is formulated in terms of the gain of the Rician $K$-factor, the reduction of the root-mean-squared delay spread, and the gain of the signalto-noise ratio at the receiver for Rician fading channels in multipath environments. The analysis is based on a double-directional channel model. For the analytical formulation, the joint channel spectrum is assumed to be decomposable into separate spectra in time and angular domains. By way of illustration, closed-form expressions for the impact of hypothetical cosine-shaped antenna patterns and conventional beamformers are derived for channels with uniform angular spectra and an exponential decaying delay spectrum. The impact factors are explicitly related to the antenna beamwidth and the number of antenna elements. In addition, the effect of misalignment between the antenna main beam and the direct path is included in the analysis. The quantitative analysis given in this paper is important for radio system design, particularly for the design of antennas and multiantenna beamformer configurations.
\end{abstract}

Index Terms-Conventional beamforming, double-directional channel, half-power beamwidth (HPBW), multiantenna beamforming, Rician $K$-factor, root-mean-squared (rms) delay spread (RDS).

\section{INTRODUCTION}

$\mathbf{I}$ $\mathrm{T}$ IS WELL known that applying directional antennas in wireless communication systems increases the signal power level in the receiver, reduces the multipath dispersive effect, and reduces the cochannel interference from other users [1], [2], [6]-[8]. An alternative but more flexible way is to use multiple antennas for directional beamforming. In particular, for wideband radio systems, such as the multigigabit-per-second system deployed at the frequency band of around $60 \mathrm{GHz}$, multiantenna beamforming is advantageous to high-data-rate transmission, which is generally limited by a stringent link budget requirement and multipath channel dispersions, and allows a low-complexity and low-cost design of transceiver systems [9], [10]. To design such a system, it is essential to have some quantitative knowledge about the impact of directional antennas and multiantenna beamformers on the radio channel and system so that the antenna patterns and array configurations can be properly designed.

Manuscript received February 6, 2007; revised June 8, 2007 and August 3, 2007. The review of this paper was coordinated by Prof. R. Qiu.

The authors are with the Department of Electrical Engineering, Eindhoven University of Technology, 5600 MB Eindhoven, The Netherlands (e-mail: H.Yang@tue.nl; M.H.A.J.Herben@tue.nl; J.A.G.Akkermans@tue.nl; P.F.M.Smulders@tue.nl).

Digital Object Identifier 10.1109/TVT.2007.907308
Measurements and ray-tracing simulations have been reported in the literature to investigate the effects of antenna directivity on radio propagation and systems in indoor and outdoor environments at different frequency bands [6], [8], [9], [11]-[14]. In detail, the reduction of the root-mean-squared (rms) delay spread (RDS) caused by antenna directivity in urban line-of-sight (LOS) street environments was experimentally studied in [8] for different beamwidths, and the effect of main beam misalignment was observed as well. In [13], it was found that the reduction of RDS is in the range of 35\%-55\% for directional antenna configurations in comparison with the omnidirectional one in indoor LOS environments. Extensive measurements and simulations were conducted in [9], [11], [12], and [14] for indoor LOS communication at 19.37 and $60 \mathrm{GHz}$, respectively. Their results indicated that the use of fairly narrow antenna beamwidths could be accepted as an alternative to adaptive equalization or multicarrier modulation for high-data-rate transmission in indoor LOS and some nonLOS scenarios.

The theory of analyzing the effect of antennas on radio transmission was introduced in [1], in which the effect of directional antennas on the signal-level and level-crossing rates was analyzed. In [15], a general expression was derived to compute the mean effective gain of mobile antennas for Rayleigh fading channels with both vertical and horizontal polarizations taken into account. A theoretical analysis of the mean effective gain of antennas in Rician channels was conducted in [16] and [17]. Clearly, the gain analysis only involves the mutual effect between the antenna power gain pattern and the power angular distribution of the multipath waves.

However, to the best of the authors' knowledge, the theoretical analysis concerning the reduction of multipath time dispersion caused by directional antennas has not been reported in the literature. The concept of double-directional channels was proposed earlier and applied to take into account the angular information of wave propagation at both the transmitter (TX) and receiver (RX) sides for channel characterization [18], [19]. The description of double-directional channels is particularly important for systems with multiple antennas at both the TX and RX sides. This paper aims to introduce a theoretical analysis on the impact of the antenna pattern on radio transmission, including signal-to-noise ratio (SNR) gain, Rician $K$-factor gain, and RDS reduction, in double-directional Rician channels.

This paper is organized as follows. Section II describes the signal and channel models applied in this paper and formulates the antenna effect on radio transmission. The approach used in Section II will be extended to multiantenna beamforming 


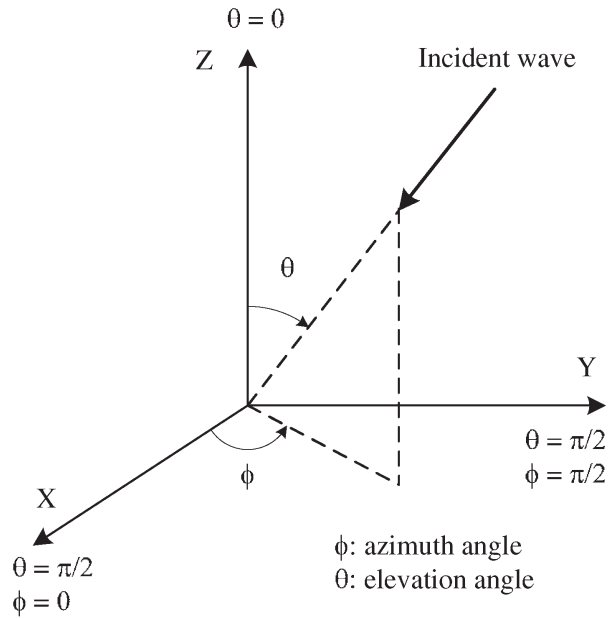

Fig. 1. Spherical coordinate system.

in Section III. Based on the channel and antenna assumptions introduced in Section IV, examples are illustrated in Section V for a hypothetical cosine-shaped antenna pattern and a conventional beamformer. Finally, conclusions are discussed in Section VI.

\section{Signal Model, Channel Characteristics, AND ANTENNA EFFECT}

Consider a radio transmission system where the signal propagation paths and the antennas at the transmitter and receiver sides are positioned in the spherical coordinate system shown in Fig. 1. Here, $\Omega$ is the coordinate point on a spherical surface given by $(\theta, \phi)$, where $\theta \in[0, \pi]$ and $\phi \in[-\pi, \pi)$ represent the elevation and azimuth angles, respectively. The direction $\Omega_{x}=\left(\theta_{x}, \phi_{x}\right)$ with $x \in\{t, r\}$ stands for the direction of the departed path at the TX side or the incident path at the RX side, respectively. Here, we assume that the same single-polarized antennas are used at the TX and RX sides. For a wideband transmission system, the baseband received signal at time $t$ can be expressed as

$$
y(t)=\sum_{n=0}^{N} h_{n} \sqrt{A_{t}\left(\Omega_{t, n}, \Psi_{t}\right) A_{r}\left(\Omega_{r, n}, \Psi_{r}\right)} \cdot s\left(t-\tau_{n}\right)+n(t)
$$

where $A_{x}\left(\Omega_{x}, \Psi_{x}\right)$ for $x \in\{t, r\}$ are the TX and RX antenna power patterns with $\Psi_{x}=\left(v_{x}, \psi_{x}\right)$ as the direction of the antenna main lobe, $s(t)$ is the baseband transmitted signal, and $n(t)$ is the additive white Gaussian noise (AWGN). The transmit power is $E\left\{|s(t)|^{2}\right\}=E_{s}$, and the noise power is $E\left\{|n(t)|^{2}\right\}=N_{0}$, within the receiver bandwidth, where $E\{\cdot\}$ denotes an expectation operation. The channel parameters $\left\{N, h_{n}, \tau_{n}, \Omega_{t, n}, \Omega_{r, n}\right\}$ are the random channel variables, i.e., the number of scattered paths, the complex amplitude, the time of arrival (TOA), the direction of departure (DOD), and the direction of arrival (DOA) of the $n$th multipath wave, respectively. The channel parameters of the LOS wave are $\left\{h_{0}, \tau_{0}, \Omega_{t, 0}, \Omega_{r, 0}\right\}$, where $\Omega_{x, 0}=\left(\theta_{x, 0}, \phi_{x, 0}\right)$.
It should be noticed that for physical channels, the channel parameters in (1) are in general randomly time-varying variables because of the arbitrary movements of the transmitter, receiver, or surrounding objects. In practice, it is reasonable to assume that the channel statistic is stationary or quasi-static, i.e., wide-sense stationary (WSS), within the time duration of one transmitted symbol or one data package. For this reason, the time dependency of the channel parameters has been omitted in (1). Moreover, signals coming via different paths will experience uncorrelated attenuations and time delays, which are referred to as uncorrelated scattering (US). The assumption of WSS and US (WSSUS) on physical channels has been experimentally confirmed and widely accepted in the literature [2]-[5]. In the rest of this paper, time-invariant channels in a local area will be considered under the WSSUS assumption.

\section{A. Double-Directional Channel Model Without Antenna Effect}

From the received signal model (1), a double-directional channel model can be retrieved to describe the channel behavior in the time, DOD, and DOA domains. For the channel configured with isotropic antennas at both TX and RX sides, the instantaneous delay-DOD-DOA channel function and the instantaneous power delay-DOD-DOA spectrum, respectively, can be written as

$$
\begin{aligned}
h\left(\tau, \Omega_{t}, \Omega_{r}\right)= & \sum_{n=0}^{N} h_{n} \delta\left(\tau-\tau_{n}\right) \\
& \times \delta\left(\Omega_{t}-\Omega_{t, n}\right) \delta\left(\Omega_{r}-\Omega_{r, n}\right) \\
P_{I}\left(\tau, \Omega_{t}, \Omega_{r}\right)= & \sum_{n=0}^{N}\left|h_{n}\right|^{2} \delta\left(\tau-\tau_{n}\right) \\
& \times \delta\left(\Omega_{t}-\Omega_{t, n}\right) \delta\left(\Omega_{r}-\Omega_{r, n}\right) .
\end{aligned}
$$

Under the WSSUS assumption, the local-mean power delay-DOD-DOA spectrum can be obtained by taking the average over the instantaneous power spectra in a local area and can be expressed by [19], [20]

$$
\begin{aligned}
P\left(\tau, \Omega_{t}, \Omega_{r}\right)= & E\left\{P_{I}\left(\tau, \Omega_{t}, \Omega_{r}\right)\right\} \\
= & E\left\{\left|h_{0}\right|^{2}\right\} \delta(\tau) \delta\left(\Omega_{t}-\Omega_{t, 0}\right) \delta\left(\Omega_{r}-\Omega_{r, 0}\right) \\
& +P_{S}\left(\tau, \Omega_{t}, \Omega_{r}\right)
\end{aligned}
$$

where

$$
\begin{aligned}
& P_{S}\left(\tau, \Omega_{t}, \Omega_{r}\right) \\
& =E\left\{\sum_{n=1}^{N}\left|h_{n}\right|^{2} \delta\left(\tau-\tau_{n}\right) \delta\left(\Omega_{t}-\Omega_{t, n}\right) \delta\left(\Omega_{r}-\Omega_{r, n}\right)\right\}
\end{aligned}
$$

is the power spectrum caused by scattered multipath waves. For convenience, the TOA of the LOS path was set to be $\tau_{0}=0$ in (4). Notice that $P(\cdot)$ and $P_{S}(\cdot)$ denote the channel power spectrum with and without the LOS path, respectively, for isotropic antenna patterns. The joint and separate spectra should be distinguished according to the parameters within $(\cdot)$. 
Later, similar notations $P^{\prime}(\cdot)$ and $P_{S}^{\prime}(\cdot)$ will be introduced to represent the channel spectra for nonisotropic antenna patterns.

Next, the power delay spectrum (PDS, or power delay profile) and the power DOD-DOA spectrum, respectively, can be derived from $P\left(\tau, \Omega_{t}, \Omega_{r}\right)$ according to

$$
\begin{aligned}
P(\tau) & =\oint \oint P\left(\tau, \Omega_{t}, \Omega_{r}\right) d \Omega_{t} d \Omega_{r} \\
& =E\left\{\left|h_{0}\right|^{2}\right\} \delta(\tau)+\underbrace{\oint \oint P_{S}\left(\tau, \Omega_{t}, \Omega_{r}\right) d \Omega_{t} d \Omega_{r}}_{P_{S}(\tau)}
\end{aligned}
$$

$$
\begin{aligned}
P\left(\Omega_{t}, \Omega_{r}\right)= & \int P\left(\tau, \Omega_{t}, \Omega_{r}\right) d \tau \\
= & E\left\{\left|h_{0}\right|^{2}\right\} \delta\left(\Omega_{t}-\Omega_{t, 0}\right) \delta\left(\Omega_{r}-\Omega_{r, 0}\right) \\
& +\underbrace{\int P_{S}\left(\tau, \Omega_{t}, \Omega_{r}\right) d \tau}_{P_{S}\left(\Omega_{t}, \Omega_{r}\right)}
\end{aligned}
$$

where $d \Omega=\sin (\theta) d \theta d \phi$ is a solid angle, and $P_{S}(\tau)$ and $P_{S}\left(\Omega_{t}, \Omega_{r}\right)$ are the PDS and DOD-DOA spectrum of the scattered waves, respectively. In addition, the separate power DOD and DOA spectra, respectively, can be defined by

$$
\begin{aligned}
& P_{S}\left(\Omega_{t}\right)=\oint P_{S}\left(\Omega_{t}, \Omega_{r}\right) d \Omega_{r} \\
& P_{S}\left(\Omega_{r}\right)=\oint P_{S}\left(\Omega_{t}, \Omega_{r}\right) d \Omega_{t} .
\end{aligned}
$$

If the total power of the channel is normalized, i.e.,

$$
\sum_{n=0}^{N} E\left\{\left|h_{n}\right|^{2}\right\}=1
$$

then the SNR in the receiver is $\rho=\left(E_{s} / N_{0}\right)$ when isotropic antennas are applied and the following equations are valid:

$$
\begin{aligned}
\oint \oint \int P\left(\tau, \Omega_{t}, \Omega_{r}\right) d \tau d \Omega_{t} d \Omega_{r} & =\int P(\tau) d \tau \\
& =\oint \oint P\left(\Omega_{t}, \Omega_{r}\right) d \Omega_{t} d \Omega_{r} \\
& =\oint P\left(\Omega_{x}\right) d \Omega_{x} \\
& =1 \\
\oint \oint \int P_{S}\left(\tau, \Omega_{t}, \Omega_{r}\right) d \tau d \Omega_{t} d \Omega_{r} & =\int P_{S}(\tau) d \tau \\
& =\oint \oint P_{S}\left(\Omega_{t}, \Omega_{r}\right) d \Omega_{t} d \Omega_{r} \\
& =\oint P_{S}\left(\Omega_{x}\right) d \Omega_{x} \\
& =\frac{1}{K+1} .
\end{aligned}
$$

Here, $1 /(K+1)$ is the power of the scattered waves, and $K$ is the ratio between the average powers contributed by the LOS path and the scattered paths, i.e.,

$$
K=\frac{E\left\{\left|h_{0}\right|^{2}\right\}}{E\left\{\sum_{n=1}^{N}\left|h_{n}\right|^{2}\right\}} .
$$

The parameter $K$ is usually called the Rician $K$-factor and is used to characterize the Rician fading channel. In addition, the RDS of the channel is calculated by

$$
\begin{aligned}
\sigma_{\tau} & =\sqrt{\frac{\int \tau^{2} P(\tau) d \tau}{\int P(\tau) d \tau}-\left(\frac{\int \tau P(\tau) d \tau}{\int P(\tau) d \tau}\right)^{2}} \\
& =\sqrt{\overline{\tau^{2}}-\bar{\tau}^{2}}
\end{aligned}
$$

where $\bar{\tau}=\left(\int \tau P(\tau) d \tau / \int P(\tau) d \tau\right)$ is the mean excess delay, and $\overline{\tau^{2}}=\left(\int \tau^{2} P(\tau) d \tau / \int P(\tau) d \tau\right)$ is the second moment of the delay spectrum $P(\tau)$. The RDS $\sigma_{\tau}$ is generally used to characterize the time dispersion of the channel.

\section{B. Impact of Antenna Pattern on Radio Channel}

When nonisotropic antennas are applied in the channel, the joint power delay-DOD-DOA spectrum becomes $P\left(\tau, \Omega_{t}, \Omega_{r}\right) A_{t}\left(\Omega_{t}, \Psi_{t}\right) A_{r}\left(\Omega_{r}, \Psi_{r}\right)$, and the separate spectra can be accordingly obtained. In particular, the PDS becomes

$$
\begin{aligned}
P^{\prime}(\tau) & =\oint \oint P\left(\tau, \Omega_{t}, \Omega_{r}\right) A_{t}\left(\Omega_{t}, \Psi_{t}\right) A_{r}\left(\Omega_{r}, \Psi_{r}\right) d \Omega_{t} d \Omega_{r} \\
& =E\left\{\left|h_{0}\right|^{2}\right\} A_{t}\left(\Omega_{t, 0}, \Psi_{t}\right) A_{r}\left(\Omega_{r, 0}, \Psi_{r}\right) \delta(\tau)+P_{S}^{\prime}(\tau)
\end{aligned}
$$

where the delay spectrum of scattered waves is

$$
P_{S}^{\prime}(\tau)=\oint \oint P_{S}\left(\tau, \Omega_{t}, \Omega_{r}\right) \cdot A_{t}\left(\Omega_{t}, \Psi_{t}\right) A_{r}\left(\Omega_{r}, \Psi_{r}\right) d \Omega_{t} d \Omega_{r}
$$

An explicit expression of $P_{S}^{\prime}(\tau)$ can be derived when the antenna patterns and the power delay angular spectrum $P_{S}\left(\tau, \Omega_{t}, \Omega_{r}\right)$ are given.

To investigate the impact of antenna patterns on the transmission system and channel, we consider the change of the Rician $K$-factor, the RDS, and the change of the SNR caused by nonisotropic antenna patterns. In specific, the following parameters are defined for the purpose of analysis: the gain of the Rician $K$-factor

$$
G_{K}=\frac{K^{\prime}}{K}
$$

the gain of the SNR

$$
G_{\rho}=\frac{\rho^{\prime}}{\rho}
$$


where $\rho$ stands for the SNR in the receiver, and the relative reduction of RDS

$$
R_{\sigma_{\tau}}=1-\frac{\sigma_{\tau}^{\prime}}{\sigma_{\tau}}
$$

Here, the two parameter sets $\left\{K, \rho, \sigma_{\tau}\right\}$ and $\left\{K^{\prime}, \rho^{\prime}, \sigma_{\tau}^{\prime}\right\}$ are for the channels configured with isotropic and nonisotropic antennas, respectively. These parameters are defined to formulate the impact of antenna patterns on propagation channels and, thus, are useful for the purpose of system design.

Here, we derive explicit expressions of the impact parameters. Notice that when nonisotropic antenna patterns are used in the channel, the channel power of the LOS path $(K /(K+1))$ is scaled by the TX and RX antenna pattern gains $A_{t}\left(\Omega_{t, 0}, \Psi_{t}\right)$ and $A_{r}\left(\Omega_{r, 0}, \Psi_{r}\right)$ along the LOS direction $\Omega_{x, 0}=\left(\theta_{x, 0}, \phi_{x, 0}\right)$. Further, the power angular spectrum of scattered waves becomes

$$
P_{S}^{\prime}\left(\Omega_{t}, \Omega_{r}\right)=P_{S}\left(\Omega_{t}, \Omega_{r}\right) A_{t}\left(\Omega_{t}, \Psi_{t}\right) A_{r}\left(\Omega_{r}, \Psi_{r}\right)
$$

which can be integrated to obtain the power of the scattered waves. Consequently, the power gain of the scattered waves due to TX-RX antenna patterns can be derived as

$E_{A}=(K+1) \oint \oint P_{S}\left(\Omega_{t}, \Omega_{r}\right) \cdot A_{t}\left(\Omega_{t}, \Psi_{t}\right) A_{r}\left(\Omega_{r}, \Psi_{r}\right) d \Omega_{t} d \Omega_{r}$

Now the Rician $K$-factor gain and the SNR gain can be readily obtained as

$$
\begin{aligned}
G_{K} & =\frac{A_{t}\left(\Omega_{t, 0}, \Psi_{t}\right) A_{r}\left(\Omega_{r, 0}, \Psi_{r}\right)}{E_{A}} \\
G_{\rho} & =\frac{K}{K+1} A_{t}\left(\Omega_{t, 0}, \Psi_{t}\right) A_{r}\left(\Omega_{r, 0}, \Psi_{r}\right)+\frac{1}{K+1} E_{A} \\
& =\beta E_{A}
\end{aligned}
$$

where $\beta=\left(K G_{K}+1\right) /(K+1)$. In addition, following the definition in (14), the reduction of RDS can be derived as

$$
R_{\sigma_{\tau}}=1-\sqrt{\frac{\overline{\tau^{\prime 2}}-\overline{\tau^{\prime}}}{\overline{\tau^{2}}-\bar{\tau}^{2}}}
$$

where the mean excess delay $\overline{\tau^{\prime}}=\left(\int \tau P^{\prime}(\tau) d \tau / \int P^{\prime}(\tau) d \tau\right)$, and the second moment of the PDS $P^{\prime}(\tau)$ is $\overline{\tau^{\prime 2}}=$ $\left(\int \tau^{2} P^{\prime}(\tau) d \tau / \int P^{\prime}(\tau) d \tau\right)$.

Clearly, for a certain power delay-DOD-DOA spectrum and antenna patterns, the impact of nonisotropic antennas on the channel can be analytically studied. Notice that the Rician $K$-factor gain and the SNR gain are independent of the PDS, and for a fixed orientation of the antenna, the SNR gain $G_{\rho}$ only depends on the $K$-factor and the gain of the scattered waves. In addition, the RDS reduction is determined by the first and second moments of the power delay spectra before and after nonisotropic antennas are applied. These moments are related to the $K$-factor and the parameter $E_{A}$.

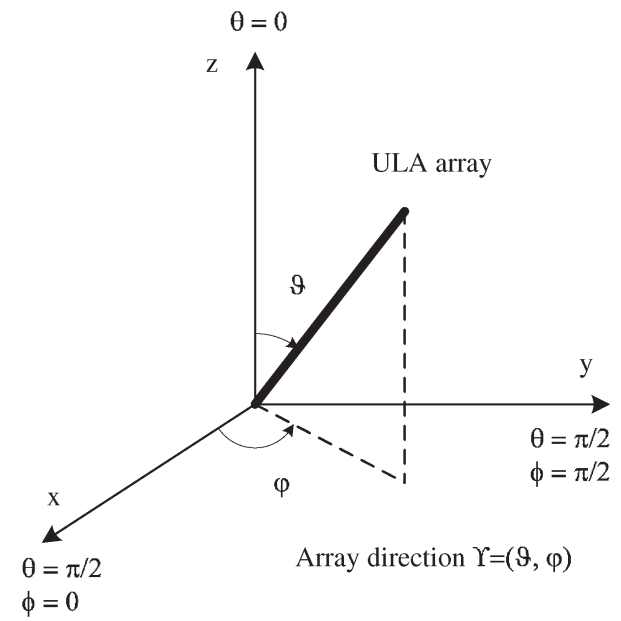

Fig. 2. ULA antenna array in the coordinate system. Each element has the same orientation, and the main lobes are perpendicular to the array direction.

\section{Extension to Multiantenna BeAmForming}

The purpose of applying directional antennas in many applications is to satisfy the link budget requirement in the receiver and, at the same time, to reduce the multipath effect on data transmission. However, besides the involvement of adjusting the main beam to a certain direction, the antenna pattern beamwidth cannot be designed as narrow as we like due to the finite size of the antenna, which results in a reduced directivity. In comparison, multiple antennas can be applied to adaptively form a desired beam pattern having its maximum gain along the desired direction. In this regard, adaptive multiantenna beamforming becomes a better solution to increase the mobility of a transceiver system, to further increase the directivity, and to reduce the multipath effect. To this end, this section will focus on the impact of multiantenna beamforming on radio transmission.

\section{A. MIMO Channel Model}

Without losing generality, here we consider the uniform linear arrays (ULAs) used at the transmitter and receiver sides. The antenna arrays are positioned in the same coordinate system as shown in Fig. 1. The first element is positioned at the origin, and the array direction is represented by $\Upsilon_{x}=\left(\vartheta_{x}, \varphi_{x}\right)$ with $x \in\{t, r\}$, as shown in Fig. 2. Assuming locally plane waves at the transmitter and receiver, the $n$th multipath wave propagation between any pair of transmitting and receiving elements can be modeled to have experienced the same amplitude attenuation but different phases due to path length differences.

For the antenna arrays composed of $P$ antenna elements and $Q$ antenna elements at the TX and RX sides, respectively, the multipath multiply-input-multiple-outout (MIMO) channel response matrix can be described by

$$
\begin{aligned}
& \mathbf{H}\left(\tau, \Omega_{t}, \Omega_{r}\right)=\sum_{n=0}^{N} \mathbf{H}_{n} \delta\left(\tau-\tau_{n}\right) \\
& \cdot \delta\left(\Omega_{t}-\Omega_{t, n}\right) \delta\left(\Omega_{r}-\Omega_{r, n}\right)
\end{aligned}
$$


where

$$
\mathbf{H}_{n}=h_{n} \mathbf{a}_{r}\left(\Omega_{r, n}, \Upsilon_{r}\right) \mathbf{a}_{t}^{H}\left(\Omega_{t, n}, \Upsilon_{t}\right)
$$

is the channel matrix of the $n$th path, the superscript ${ }^{H}$ represents the Hermitian operation, and $\mathbf{a}_{x}=\mathbf{a}_{x}\left(\Omega_{x, n}, \Upsilon_{x}\right)$ is the array response vector of the direction $\Omega_{x, n}$. The array response at either the transmitter or receiver side can be expressed by

$$
\mathbf{a}(\Omega, \Upsilon)=\left[1 e^{j \varepsilon} \cdots e^{j(M-1) \varepsilon}\right]^{T}
$$

where the superscript ${ }^{T}$ denotes the transpose operation, the relative phase difference between elements

$$
\varepsilon=\frac{2 \pi d}{\lambda}(\sin \theta \sin \vartheta \cos [\phi-\varphi]+\cos \theta \cos \vartheta)
$$

$\lambda$ is the wavelength, $d$ is the antenna element spacing, and $M \in$ $\{P, Q\}$ is the number of elements. Here, for convenience, the subscript $x \in\{t, r\}$ was omitted for $\mathbf{a}_{x}, \Omega_{x}$, and $\Upsilon_{x}$.

\section{B. Multiantenna Beamforming}

Suppose that ULA arrays with nonisotropic elements are applied, and a narrowband beamforming is performed at the TX and RX sides. All the elements are assumed to have the same orientation, i.e., $\Psi_{x}$ is the same for all the elements at the TX or RX side. Therefore, the weighted output of the beamformer in the receiver is written as

$$
\begin{aligned}
y^{\prime}(t)=\sum_{n=0}^{N} h_{n} & \underbrace{\sqrt{A_{r}\left(\Omega_{r, n}, \Psi_{r}\right)} \mathbf{w}_{r}^{T} \mathbf{a}_{r}}_{\sqrt{C_{r}\left(\Omega_{r, n}, \Psi_{r}, \Upsilon_{r}\right)}} \\
& \underbrace{\sqrt{A_{t}\left(\Omega_{t, n}, \Psi_{t}\right)} \mathbf{a}_{t}^{H} \mathbf{w}_{t}}_{\sqrt{C_{t}\left(\Omega_{t, n}, \Psi_{t}, \Upsilon_{t}\right)}} s\left(t-\tau_{n}\right)+\mathbf{w}_{r}^{T} \mathbf{n}
\end{aligned}
$$

where the total transmit power is equally allocated to each element, i.e., $E\left\{|s(t)|^{2}\right\}=\left(E_{s} / P\right)$, the weights satisfy $\mathbf{w}_{t}^{H} \mathbf{w}_{t}=P$ and $\mathbf{w}_{r}^{H} \mathbf{w}_{r}=Q$, and the elements of the noise vector $\mathbf{n}=\left[n_{1}(t), n_{2}(t), \cdots, n_{Q}(t)\right]^{T}$ in the receiver are independently and identically distributed AWGN with variance $N_{0}$. The phase information of $\mathbf{w}_{r}^{T} \mathbf{a}_{r}$ and $\mathbf{a}_{t}^{H} \mathbf{w}_{t}$ has been included in the channel impulse response $h_{n}$, which will not affect the following derivations. The synthesized power pattern $C$ is merely the product of the antenna pattern $A$ and the array pattern $B$, i.e.,

$$
C(\Omega, \Psi, \Upsilon)=A(\Omega, \Psi) B(\Omega, \Upsilon)
$$

where the array pattern is written as

$$
B(\Omega, \Upsilon)=\left|\mathbf{a}^{H} \mathbf{w}\right|^{2} .
$$

Notice that the signal model (29) is exactly the same as the model (1) of the single-antenna case by replacing the antenna pattern $A$ by the synthesized pattern $C$. Therefore, the joint impact of the element pattern and the multiantenna beamforming on the channel can be analyzed by the same approach as in Section II-B. Keep in mind that the computation of impact factors here is always relative to the case of the singleinput-single-output channel configured with isotropic antennas. Particularly, the $K$-factor gain $G_{K}$ and the RDS reduction $G_{\sigma_{\tau}}$ may be obtained by simply replacing the antenna pattern $A_{x}\left(\Omega_{x, n}, \Psi_{x}\right)$ by the synthesized pattern $C_{x}\left(\Omega_{x, n}, \Psi_{x}, \Upsilon_{x}\right)$ into (22) and (24), respectively. However, the expression of the SNR gain here is somewhat different. Bearing in mind that the transmit power in each element is $1 / P$ times the total transmit power and that the total receiver noise is $Q$ times the noise power at each element, the SNR gain $G_{\rho}=\left(\rho^{\prime} / \rho\right)$ is calculated as

$$
\begin{aligned}
G_{\rho} & =\frac{\frac{E_{S}}{P} \cdot\left(\frac{K}{K+1} C_{t}\left(\Omega_{t, 0}, \Psi_{t}, \Upsilon_{t}\right) C_{r}\left(\Omega_{r, 0}, \Psi_{r}, \Upsilon_{r}\right)+\frac{1}{K+1} E_{C}\right)}{Q N_{0} \cdot \rho} \\
& =\frac{\beta E_{C}}{P Q}
\end{aligned}
$$

where $\rho=\left(E_{S} / N_{0}\right)$, and $\beta=\left(K G_{K}+1\right) /(K+1)$. The Rician $K$-factor gain is given by

$$
G_{K}=\frac{C_{t}\left(\Omega_{t, 0}, \Psi_{t}, \Upsilon_{t}\right) C_{r}\left(\Omega_{r, 0}, \Psi_{r}, \Upsilon_{r}\right)}{E_{C}}
$$

and the power gain of the scattered waves $E_{C}$ is given by

$$
\begin{aligned}
E_{C}=(K+1) \oint \oint P_{S}\left(\Omega_{t}, \Omega_{r}\right) C_{t}\left(\Omega_{t}, \Psi_{t}, \Upsilon_{t}\right) \\
\cdot C_{r}\left(\Omega_{r}, \Psi_{r}, \Upsilon_{r}\right) d \Omega_{t} d \Omega_{r}
\end{aligned}
$$

for the synthesized pattern. Note that the results in Section II-B for single TX and RX elements are merely a special case in this section.

\section{Assumptions on Channel And Antenna}

As shown in Section II, by knowing the power distributions of radio waves in the time and angular domains, the impact of directional antennas and multiantenna beamformers on the channel can be analyzed. When isotropic antennas are used, the power distributions of radio waves will be very dependent on the propagation environment. Many researchers have conducted channel measurements to study the joint delay-angular spectra in certain environments [21]-[25]. The measured delay-angular spectra can be applied to study the impact of antennas on channels. Moreover, statistical models for the joint channel power spectrum $P\left(\tau, \Omega_{t}, \Omega_{r}\right)$ can be applied as an input to study the impact of antennas and beamformers. However, it is arduous to find a general form or an explicit function for describing the joint multidimensional information of radio channels. In this regard, the integration in (16) is not a trivial task for the purpose of analytical formulation. To solve this limitation, a general approach is to assume that the joint power delay-angular spectrum can be decomposed into separate spectra in the time and angular domains [22], [23]. Based on the decomposition, the integration in (16) could be relaxed, as shown in Section V, since statistical models for separate delay spectra and angular spectra have been widely studied and are available in the literature. 


\section{A. Separability of Joint Spectrum in a Single Cluster Model}

Assuming that the joint power spectrum $P_{S}\left(\tau, \Omega_{t}, \Omega_{r}\right)$ of the scattered waves is densely distributed in delay and angles, and that the joint spectrum is proportional to the angular spectrum at a specific time delay and proportional to the delay spectrum at a specific direction [22], i.e.,

$\left.\left.P_{S}\left(\tau, \Omega_{t}, \Omega_{r}\right)\right|_{\tau} \propto P_{S}\left(\Omega_{t}, \Omega_{r}\right) P_{S}\left(\tau, \Omega_{t}, \Omega_{r}\right)\right|_{\left(\Omega_{t}, \Omega_{r}\right)} \propto P_{S}(\tau)$

then the spectrum can be decomposed as the product of delay spectrum and angular spectrum as

$$
\text { Decomposition } 1: P_{S}\left(\tau, \Omega_{t}, \Omega_{r}\right)=c_{1} P_{S}(\tau) P_{S}\left(\Omega_{t}, \Omega_{r}\right)
$$

where the constant $c_{1}=K+1$ can be determined from (6)-(12). The decomposition has been experimentally validated for typical outdoor urban channel environments in [22]. In indoor environments, scattered waves become even denser in the DOD and DOA, and thus, the decomposition could be valid as well. In addition, it is reasonable to assume that the angular spectra of the DOD and DOA of scattered waves are independent of each other. This leads to the following decomposition:

$$
\text { Decomposition } 2: P_{S}\left(\Omega_{t}, \Omega_{r}\right)=c_{2} P_{S}\left(\Omega_{t}\right) P_{S}\left(\Omega_{r}\right)
$$

where the constant $c_{2}=K+1$. Combining (36) and (37) leads to the decomposition

$$
P_{S}\left(\tau, \Omega_{t}, \Omega_{r}\right)=(K+1)^{2} P_{S}(\tau) P_{S}\left(\Omega_{t}\right) P_{S}\left(\Omega_{r}\right) .
$$

With the decomposition in (38), the delay spectrum of scattered waves in (16) becomes

$$
P_{S}^{\prime}(\tau)=F_{t, C} F_{r, C} P_{S}(\tau)
$$

due to the synthesized pattern (30). Here, the total gain of scattered waves $E_{C}=F_{t, C} F_{r, C}$ is the product of the gains separately contributed by the synthesized patterns at the TX and RX sides, where

$$
F_{x, C}=(K+1) \oint P_{S}\left(\Omega_{x}\right) C_{x}\left(\Omega_{x}, \Psi_{x}, \Upsilon_{x}\right) d \Omega_{x} .
$$

Taking the separate spectra into (33), (32), and (24), the impact of antennas and beamformer on channels can be analytically obtained.

\section{B. Power Distribution in Angular Domain}

If the scattered waves are densely and uniformly distributed in the angular region

$$
U_{x}=\left(\theta_{x} \in\left[\theta_{x}^{L}, \theta_{x}^{H}\right], \phi_{x} \in\left[\phi_{x}^{L}, \phi_{x}^{H}\right]\right)
$$

with $x \in\{t, r\}$ for the DOD and DOA, respectively, then the angular spectra of the scattered waves can be expressed by

$$
P_{S}\left(\Omega_{x}\right)= \begin{cases}\frac{1}{(K+1)\left(\phi_{x}^{H}-\phi_{x}^{L}\right)\left(\cos \theta_{x}^{L}-\cos \theta_{x}^{H}\right)}, & \Omega_{x} \in U_{x} \\ 0, & \text { others. }\end{cases}
$$

Now, the gain of the scattered waves becomes

$$
F_{x, C}=\frac{\oint_{U_{x}} C_{x}\left(\Omega_{x}, \Psi_{x}, \Upsilon_{x}\right) d \Omega_{x}}{\left(\phi_{x}^{H}-\phi_{x}^{L}\right)\left(\cos \theta_{x}^{L}-\cos \theta_{x}^{H}\right)}
$$

which is a constant for the waves distributed in a certain region. In the following, two special cases are presented for uniform angular distribution.

1) Uniform Angular Distribution in a Sphere: The channel power of the scattered waves is uniformly distributed in a sphere for DOD and DOA, i.e., $U_{x}=\left(\theta_{x} \in[0, \pi], \phi_{x} \in[-\pi, \pi)\right)$. Then, the gain of the scattered waves caused by the synthesized pattern (30) becomes

$$
F_{x, C}=\frac{1}{4 \pi} \oint C_{x}\left(\Omega_{x}, \Psi_{x}, \Upsilon_{x}\right) d \Omega_{x}
$$

In particular, the gain caused by an antenna pattern is $F_{x, A}=1$, which is independent of the orientation and type of antenna, since the antenna pattern always satisfies $\oint A_{x}\left(\Omega_{x}, \Psi_{x}\right) d \Omega_{x}=4 \pi$.

2) Uniform Angular Distribution in the Azimuth Plane: The power of the scattered waves is uniformly distributed in the azimuth plane, i.e., $U_{x}=\left(\theta_{x} \rightarrow(\pi / 2), \phi_{x} \in[-\pi, \pi)\right)$. The gain of the scattered waves caused by the synthesized pattern (30) can be obtained by

$$
\begin{aligned}
F_{x, C} & =\lim _{\theta_{x}^{L}, \theta_{x}^{H} \rightarrow \frac{\pi}{2}} \frac{\int_{-\pi}^{\pi} \int_{\theta_{x}^{L}}^{\theta_{x}^{H}} C_{x}\left(\Omega_{x}, \Psi_{x}, \Upsilon_{x}\right) \sin \left(\theta_{x}\right) d \theta_{x} d \phi_{x}}{2 \pi\left(\cos \theta_{x}^{L}-\cos \theta_{x}^{H}\right)} \\
& =\frac{1}{2 \pi} \int_{-\pi}^{\pi} C_{x}\left(\phi_{x}, \psi_{x}, \varphi_{x}\right) d \phi_{x}
\end{aligned}
$$

where $C_{x}\left(\phi_{x}, \psi_{x}, \varphi_{x}\right)$ is the pattern in the azimuth plane. In particular, for a cosine-shaped antenna power pattern $A$ that will be introduced in (48), the gain is equal to

$$
\begin{aligned}
F_{x, A} & =\frac{1}{2 \pi} \int_{-\pi}^{\pi} 2(2 q+1) \cos ^{2 q} \phi_{x} d \phi_{x} \\
& =\frac{(2 q+1) \Gamma\left[\frac{1}{2}+q\right]}{\sqrt{\pi} \Gamma[1+q]}
\end{aligned}
$$

in case the main lobe direction is in the azimuth plane, where $\Gamma[\cdot]$ is the Gamma function.

\section{Shape of Delay Spectrum}

It can be seen from (24) that the reduction of RDS that is caused by nonisotropic antennas depends on the first and second moments of the power delay spectra before and after introducing nonisotropic antennas. Under the decomposition of (38), the delay spectrum shape of the scattered waves will not be changed by the use of nonisotropic antennas. Therefore, if the spectrum shape is known, the reduction of RDS can be readily 
computed. For an exponentially decaying shape, the PDS can be expressed by

$$
P(\tau)= \begin{cases}0, & \tau<0 \\ \frac{K}{K+1} \delta(\tau), & \tau=0 \\ P_{S}(\tau), & \tau>0\end{cases}
$$

where $P_{S}(\tau)=\gamma e^{-\gamma \tau} /(K+1)$ is the PDS of scattered waves, and $\gamma$ is the decay exponent.

\section{Power Patterns of Antenna and Beamformer}

The antenna power pattern is a 3-D representation of the power radiation properties of an antenna and is generally described by a complicated function depending on the type of antenna [26], [27]. Directivity and half-power beamwidth (HPBW) are two of the most important parameters to describe an antenna pattern. Here, we introduce the cosine-shaped power pattern of antenna elements that will be used in Section V. Throughout this paper, the applied antennas are considered to be single polarized.

1) Cosine-Shaped Antenna Pattern: For a cosine-shaped antenna pattern positioned in the spherical coordinate system (see Fig. 1) with the main lobe direction aligned with the $X$-axis, i.e., $\Psi=((\pi / 2), 0)$, the 3 -D power pattern is expressed by

$$
A(\theta, \phi)=2(2 q+1)(\sin \theta \cos \phi)^{2 q}
$$

with $\theta \in[0, \pi]$ and $\phi \in[-(\pi / 2),(\pi / 2)]$. The parameter $q \geq 0$ is used to adjust the pattern shape and can be related to the HPBW of the pattern. For such an antenna pattern, the HPBWs on the principal azimuth and elevation planes are the same and are expressed by

$$
\sigma_{A}=2 \arccos 2^{-\frac{1}{2 q}}
$$

The cosine-shaped pattern has no sidelobes but is a good approximation of the power patterns for many types of elementary antennas, such as horn, patch, and dipole antennas [27].

2) Beam Pattern of Conventional Beamformer: In Section III-B, a general approach has been described for the multiantenna beamforming of ULA arrays. For a conventional beamformer applied in the multipath MIMO channel (25), the main beams at the TX and RX sides are steered to the direction of the direct path by adjusting the phase of the weight at each antenna element. Particularly, the weight equals the conjugate of the array vector at the direction of the LOS path, i.e., $\mathbf{w}=\mathbf{a}^{*}\left(\Omega_{0}, \Upsilon\right)$. Consider ULA arrays at the TX and RX sides positioned on the $Y-Z$ plane, i.e., $\Upsilon=(\vartheta,(\pi / 2))$, and each element has the cosine-shaped power pattern with the same beamwidth and orientation. The main lobe direction of each element is parallel to the direction of the $X$-axis, i.e., $\Psi=((\pi / 2), 0)$, and then, the synthesized pattern is readily attained as

$$
C(\theta, \phi, \vartheta)=A(\theta, \phi) B(\theta, \phi, \vartheta)
$$

thanks to the symmetric feature of the antenna pattern. The array pattern is written as

$$
B(\theta, \phi, \vartheta)=\frac{\sin ^{2} \frac{M \varepsilon^{\prime}}{2}}{\sin ^{2} \frac{\varepsilon^{\prime}}{2}}
$$

where the parameter

$$
\begin{aligned}
\varepsilon^{\prime}=\frac{2 \pi d}{\lambda}\left\{\left(\sin \theta \sin \phi-\sin \theta_{0} \sin \phi_{0}\right) \sin \vartheta\right. & \\
& \left.+\left(\cos \theta-\cos \theta_{0}\right) \cos \vartheta\right\}
\end{aligned}
$$

and $\Omega_{0}=\left(\theta_{0}, \phi_{0}\right)$ is the direction of the LOS wave.

For the synthesized pattern, the directivity at the direction of the LOS path is equal to $2 M^{2}(2 q+1)\left(\sin \theta_{0} \cos \phi_{0}\right)^{2 q}$. When the LOS path shifts away from the broadside at $((\pi / 2), 0)$, the directivity at $\Omega_{0}$ is reduced because of the misalignment between the LOS path and the main lobe of each element. In addition, the beamwidth of the synthesized pattern $\sigma_{C}$ depends not only on the antenna pattern but also on the array pattern that is related to the number of elements and the positioning of the array. In practice, to have a sufficient radio coverage, the antenna pattern generally has a much wider beam than the array pattern, and in this case, the beamwidth of the synthesized pattern can be approximated by $\sigma_{C} \approx \sigma_{B}$ [28], where $\sigma_{B}$ denotes the HPBW of the array pattern $B$.

\section{ImPACT ANALYSIS AND ILLUSTRATIVE EXAMPLES}

Directivity and beamwidth are two important parameters to characterize a directional antenna and have a significant effect on the channel. Therefore, it is interesting to quantitatively relate the antenna pattern parameters with their impact on the channel. Based on the channel and antenna models in the previous section, the impact of single-antenna and multiantenna beamformers on the channels is analytically formulated and illustrated by examples in this section.

\section{A. Impact Analysis on the Channel}

Suppose that the joint channel spectrum is decomposable as in (38), and the angular spectra are uniformly distributed either in a sphere or in the azimuth plane. Consider the conventional beamforming of a ULA array with the orientation $\Upsilon=(\vartheta,(\pi / 2))$. Applying the synthesized pattern $C(\theta, \phi, \vartheta)$ in (50) to (33), (32), and (24), respectively, results in the $K$-factor gain, SNR gain, and RDS reduction of the channel due to the conventional beamforming, i.e.,

$$
\begin{aligned}
G_{K} & =\frac{P^{2} Q^{2} A\left(\theta_{t, 0}, \phi_{t, 0}\right) A\left(\theta_{r, 0}, \phi_{r, 0}\right)}{F_{t, C} F_{r, C}} \\
G_{\rho} & =\frac{\beta F_{t, C} F_{r, C}}{P Q} \\
R_{\sigma_{\tau}} & =1-\frac{1}{\beta} \sqrt{\frac{\beta \eta-1}{\eta-1}} .
\end{aligned}
$$


Here

$$
F_{x, C}=\frac{1}{4 \pi} \int_{-\pi}^{\pi} \int_{0}^{\pi} C_{x}(\theta, \phi, \vartheta) \sin \theta d \theta d \phi
$$

or

$$
F_{x, C}=\frac{1}{2 \pi} \int_{-\pi}^{\pi} C_{x}\left(\frac{\pi}{2}, \phi, \vartheta\right) d \phi
$$

are the gains of scattered waves distributed in a sphere or in the azimuth plane, respectively. The parameter

$$
\eta=\frac{\overline{\tau^{2}}}{\bar{\tau}^{2}}
$$

is the ratio between the second moment and the squared first moment of the PDS for channels with isotropic antennas. For the exponentially decaying delay spectrum in (47), the ratio $\eta=2(K+1)$.

\section{B. Illustrative Examples}

Given the Rician channel $K$-factor, the antenna pattern, and the number of elements, in the following, the statistical change of $K$-factors, SNR delay spread, and RDS in the receiver will be predicted by using (53)-(55) for the exponential decaying PDS.

1) Impact of a Single Directional Element: Consider a scenario where an isotropic antenna is applied at the transmitter side and a directional antenna is applied at the receiver side. The $K$-factor gain, the SNR gain, and the reduction of RDS are predicted and plotted in Fig. 3 versus the RX beamwidths. Here, the thick and thin lines are the results for the uniform waves distributed in a sphere or in the azimuth plane, respectively. From these figures, we have the following observations.

- When the arrival direction of the LOS path is perfectly aligned with the main lobe direction at $\Omega_{t, 0}=\left(90^{\circ}, 0^{\circ}\right)$ (solid lines), the impact factors decrease with the HPBW, which means that it is preferable to have the HPBW as small as possible. In addition, the SNR gain and the reduction of RDS are more significant for channels with a larger Rician $K$-factor.

- However, when the main lobe is misaligned with the LOS path (dash lines), e.g., $\Omega_{t, 0}=\left(90^{\circ}, 10^{\circ}\right)$, the received power can significantly drop, and for a narrow beam antenna, the power is mainly contributed by scattered waves. As a result, the $K$-factor gain and the RDS reduction can only be effectively achieved when the HPBW of the antenna beam is sufficiently large. It is also noticed from Fig. 3(b) that if the waves are concentrated in the azimuth plane (thin lines), the drop of the SNR gain caused by the misalignment does not go deeper as the beamwidth narrows because the directivity is so large that the gained power from the scattered waves exceeds the power from the LOS wave.

- For the scattered waves distributed in a sphere, the $K$-factor gain and the RDS reduction are larger than those in the azimuth plane, whereas the SNR gain is slightly

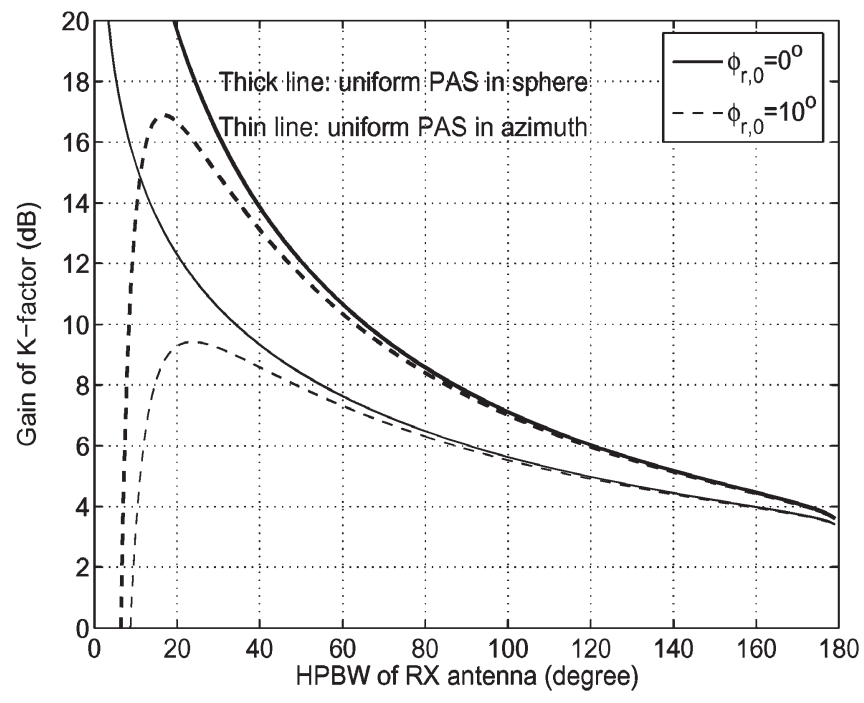

(a)

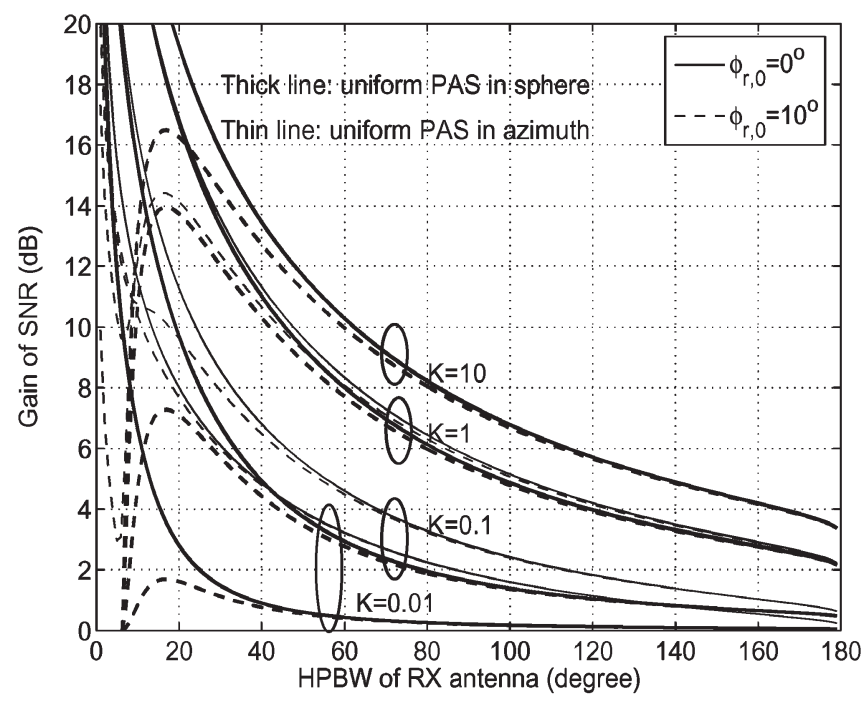

(b)

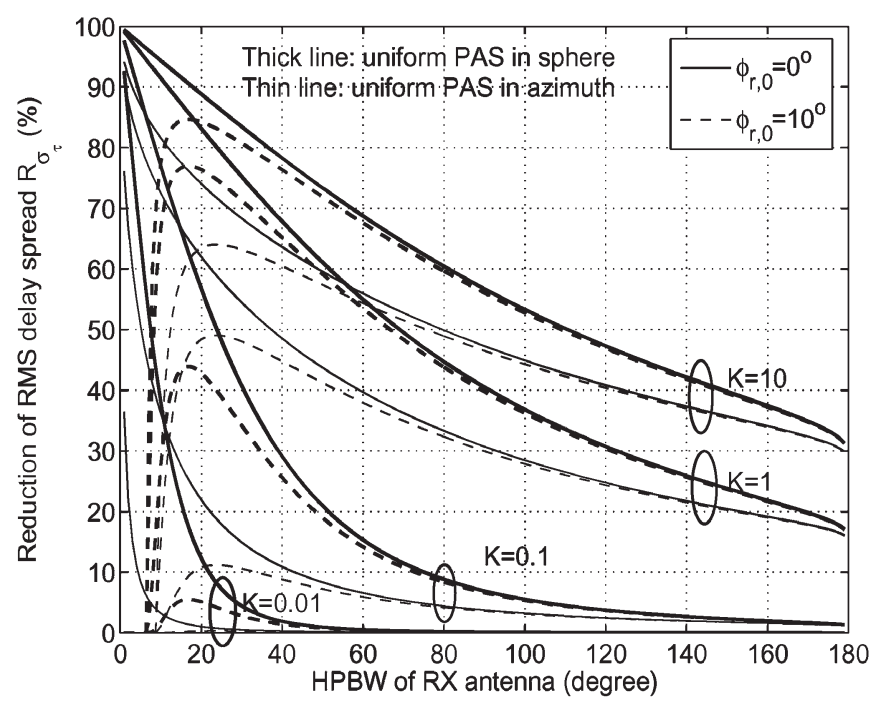

(c)

Fig. 3. With an isotropic antenna at the TX side, (a) the $K$-factor gain, (b) the SNR gain, and (c) the reduction of RDS, over the RX antenna beamwidth. The arriving directions of the LOS path considered here are $\phi_{0}=0^{\circ}$ and $10^{\circ}$. 
lower. However, as $K>1$, the SNR gain becomes less sensitive to wave distribution. The impact difference is due to the fact that the waves distributed in a sphere are suppressed in a larger extent than those in the azimuth plane.

Theoretically, the largest $K$-factor gain and RDS reduction can be achieved at a certain beamwidth for the misalignment $\phi_{0} \neq 0$ between the main lobe and the LOS path. By computing $\left(\partial G_{K} / \partial \sigma_{A}\right)=0$ and $\left(\partial R_{\sigma_{\tau}} / \partial \sigma_{A}\right)=0$, the optimum HPBW for a small misalignment $\phi_{0}$ can be approximated by

$$
\begin{aligned}
\sigma_{A[\mathrm{opt}]} & \approx 1.67 \phi_{0} \\
\sigma_{A[\mathrm{opt}]} & \approx 2.35 \phi_{0}
\end{aligned}
$$

for the scattered waves in a sphere and in the azimuth plane, respectively (see Appendix A). As for the SNR gain, the optimum SNR gain $G_{\rho}$ is achieved at $\sigma_{A} \approx 1.67 \phi_{0}$ for the case of scattered waves in a sphere, whereas for the case in the azimuth plane, the gain $G_{\rho}$ is not a strictly convex function.

2) Impact of Conventional Beamforming: When designing a multiantenna beamforming system, the requirements with regard to radio coverage and SNR gain are important issues to take into account. The radio coverage is related to the individual antenna pattern beamwidth, and the SNR gain depends not only on the directivity of elements but also on the array configuration, e.g., the number of elements. The impact factors in (53)-(55) can be taken as the criteria for selecting the antenna pattern and the number of elements.

By way of illustration, we consider a TX-RX beamforming with isotropic elements at the TX side and directional elements at the RX side. The numbers of elements are $(P, Q)=$ $(1,1),(2,2),(3,3),(4,4),(5,5)$, and $(6,6)$; the $\mathrm{TX}$ and RX arrays are positioned in the direction at $\Upsilon_{x}=\left(90^{\circ}, 90^{\circ}\right)$; and the LOS wave at the TX side is always in the direction of $\Omega_{t, 0}=\left(90^{\circ}, 0\right)$. Fig. 4 depicts the $K$-factor gain, SNR gain, and RDS reduction versus the RX antenna beamwidth for the two cases of $\Omega_{r, 0}=\left(90^{\circ}, 0^{\circ}\right)$ and $\left(90^{\circ}, 10^{\circ}\right)$, respectively. The Rician channel with $K=1$ is considered for the computation of SNR gain and RDS reduction. In addition, to show the scanning range of the array, Fig. 5 depicts the impact factors over the scanning angle $\phi_{r, 0} \in\left[-90^{\circ}, 90^{\circ}\right]$ for the RX element beamwidth $\sigma_{A}=95^{\circ}$.

For a specific design requirement, the number of elements and antenna beamwidth can be determined, and the statistical impact of this configuration on the channel can be checked from these figures. For instance, the link budget requirement of 20-dB gain in the channel with $K=1$ can be satisfied by using an antenna array with $(P, Q)=(6,6)$ with the RX element beamwidth $\sigma_{A}=95^{\circ}$ [see Fig. 4(b)]. This configuration leads to a $3-\mathrm{dB}$ scan range that is about the same as the RX element beamwidth [see Fig. 5(b)]. This observation confirms the analysis in Appendix B that a 3-dB azimuth scan range can be approximated by the element beamwidth

$$
\phi_{\text {scan }} \approx \sigma_{A}
$$

for a fairly large $K$-factor and a large number of elements. It is further observed that within the $3-\mathrm{dB}$ scan range, the $K$-factor gain is about $22.5 \mathrm{~dB}$, and the reduction of RDS is about $87.5 \%$.

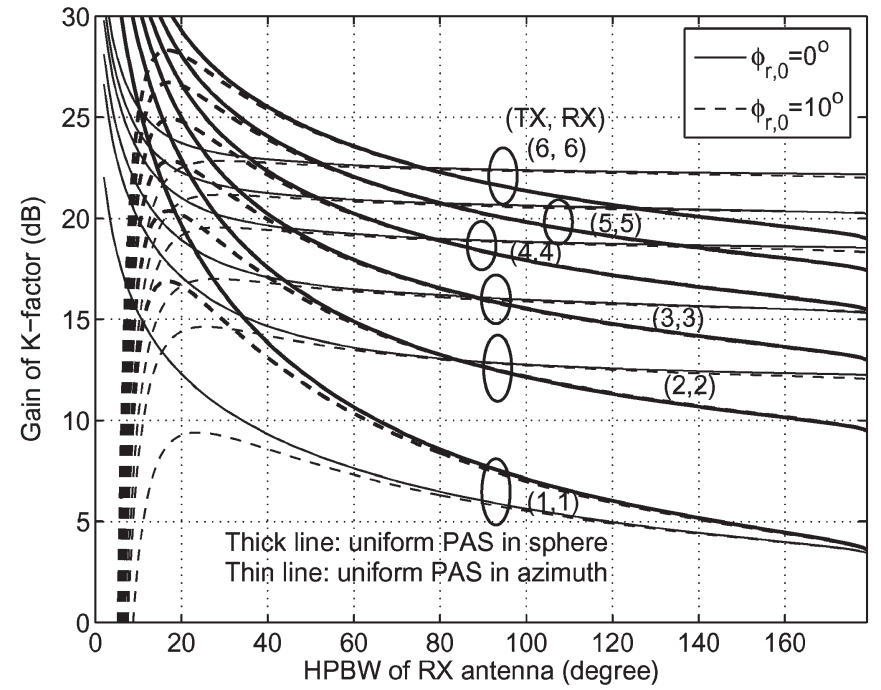

(a)

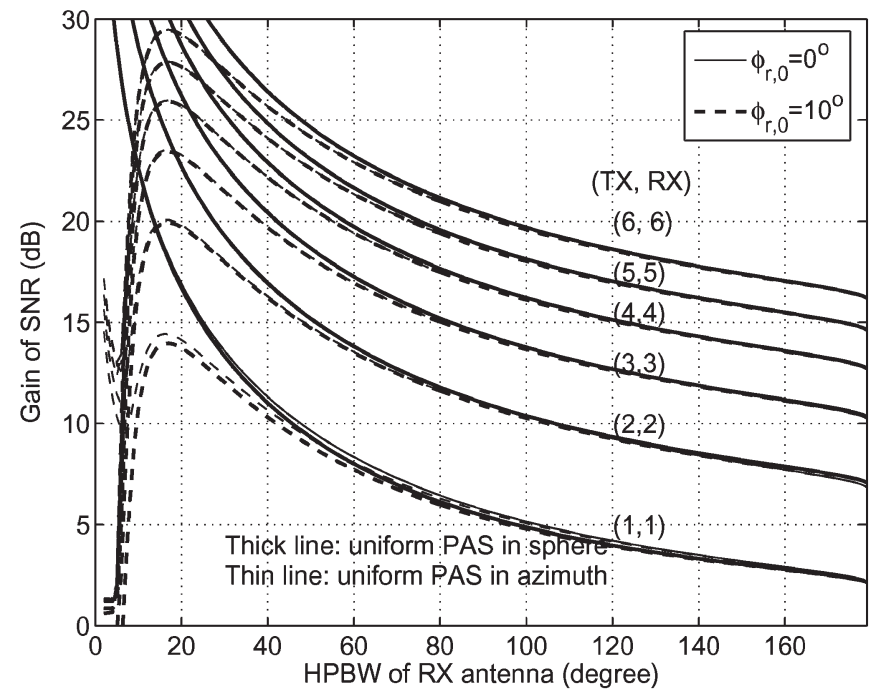

(b)

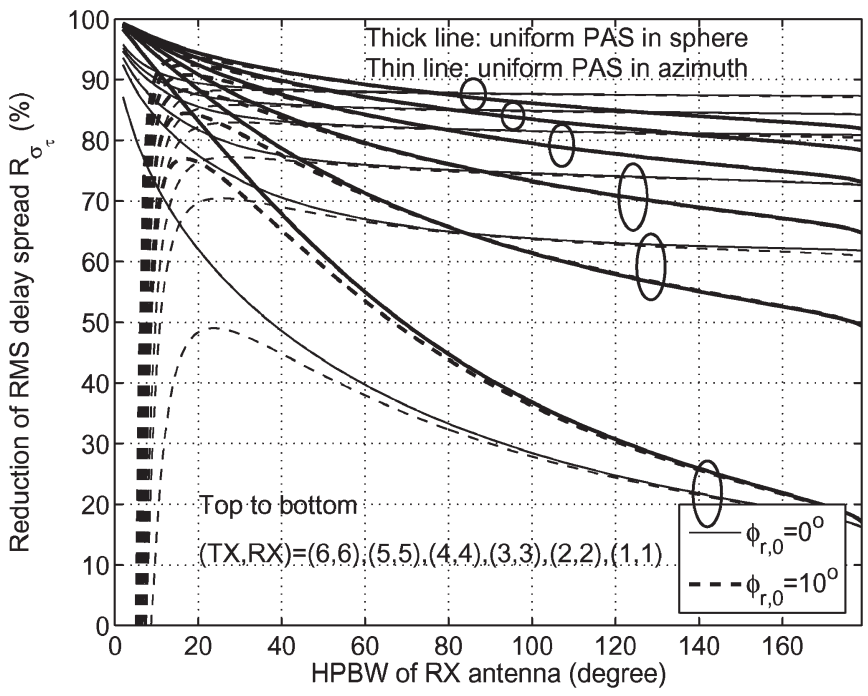

(c)

Fig. 4. For isotropic and directional antenna elements at the TX and RX sides, (a) the $K$-factor gain, (b) the SNR gain, and (c) the RDS reduction caused by conventional beamforming over the RX element beamwidth for various $(\mathrm{TX}, \mathrm{RX})=(P, Q)$ configurations. 


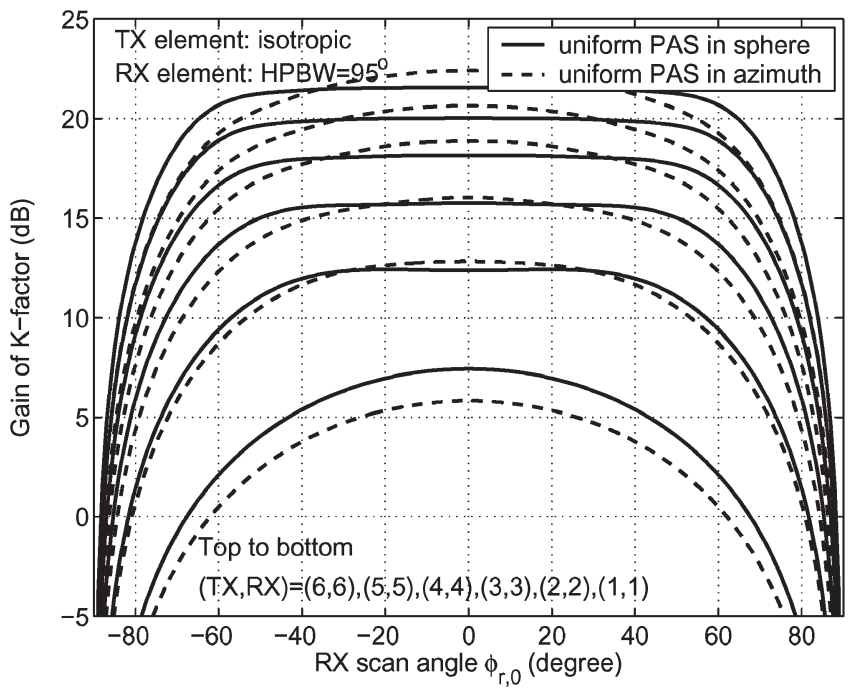

(a)

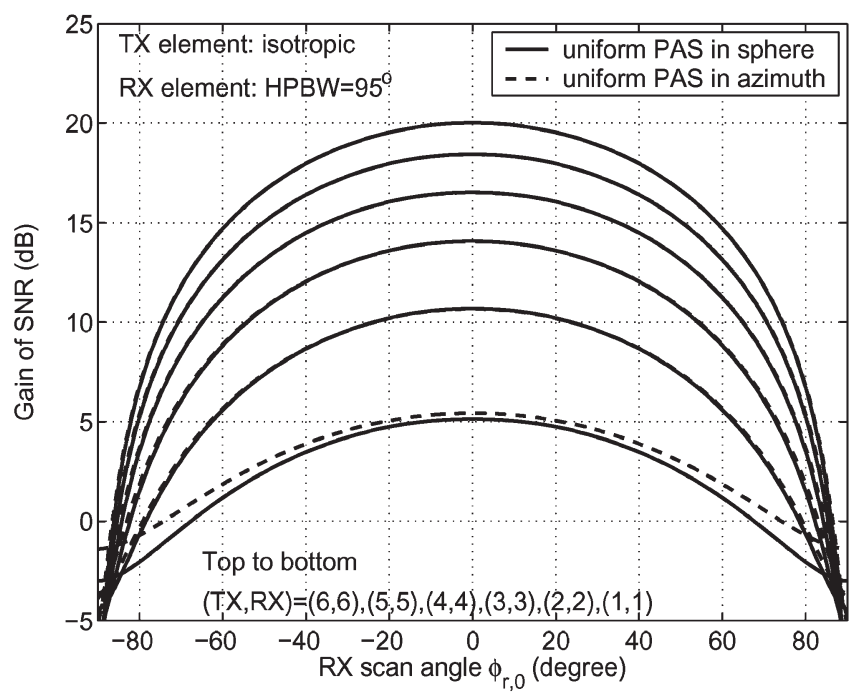

(b)

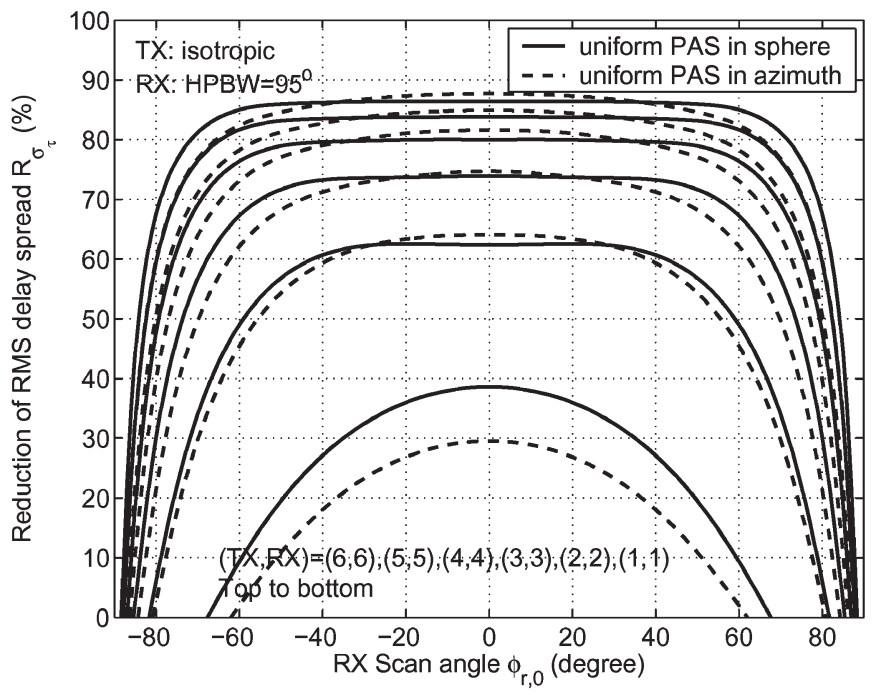

(c)

Fig. 5. For isotropic and directional antenna $\left(\sigma_{A}=95^{\circ}\right)$ elements at the TX and RX sides, (a) the $K$-factor gain, (b) the SNR gain, and (c) the RDS reduction over the RX scan angles $\phi_{r, 0}$ for various $(\mathrm{TX}, \mathrm{RX})=(P, Q)$ configurations.

\section{Discussion}

The impact of the radiation pattern on the considered channels is predicted here without taking into account some practical issues, such as antenna cross polarizations and sidelobes. When both the vertical- and horizontal-polarized field components exist in the wave transmission, a similar approach as in Section II can be used to predict the impact of antennas on the channel, but the antenna gain patterns and the channel power spectra have to be separately considered [15], [29]. The existence of sidelobes in the antenna pattern will lead to a reduced directivity, and as a result, the Rician $K$-factor gain and the RDS reduction become less effective.

It is shown in Section IV that the assumption about a decomposable channel spectrum leads to an easier analysis in this section. For further study, it is interesting to model the joint distribution of channel power in time delay and angular domain, as conducted in [30] for urban environments, and to investigate the decomposability of realistic channels. In addition, in practical propagation channels, the scattered waves are often cluster-wise distributed in time and space, and the departing and arriving directions are typically not uniformly distributed. It is yet not known how much the impact on realistic channels is different from those obtained in this section and which one of the assumptions about the exponentially decaying PDS and uniform PAS will give the most significant effect on the differences. This analysis needs to be conducted in the future.

\section{CONCLUSION}

In this paper, the impact of directional antennas and multiantenna beamformers on radio transmission has been analytically formulated for multipath Rician channel environments. By way of illustration, a hypothetical antenna with the cosineshaped power pattern was applied to show the impact on the channel with an exponential PDS and uniform power angle spectra. It was found, for instance, that in the case of misalignment between the antenna main lobe and the LOS wave, the optimal HPBW of the antenna is equal to about twice the misaligned angle. By using a directional antenna at one side of the radio link, the Rician $K$-factor and the SNR gain can range up to $16 \mathrm{~dB}$, and the RDS reduction may be more than $80 \%$. If multipath beamformers are used at both sides of the radio link, the Rician $K$-factor gain, SNR gain, and RDS reduction will be even higher. Further, it was found that for conventional beamforming, the 3-dB scan range can be approximated by the antenna element HPBW.

\section{APPENDIX A \\ Approximation OF THE OPTIMUM HPBW FOR MAIN LOBE MisaLIGNMENT}

\section{A. Uniform PAS in the Azimuth Plane}

Suppose that the scattered waves are uniformly distributed in the azimuth plane. In the case of the misalignment between the LOS path and the main lobe direction, the optimum HPBW 
concerning the largest $K$-factor and RDS reduction can be achieved by solving

$$
\psi\left[\frac{1}{2}+q\right]-\psi[1+q]=\ln \cos ^{2} \phi_{0}
$$

which is obtained by computing $\left(\partial G_{K} / \partial \sigma_{A}\right)=0$ and $\left(\partial R_{\sigma_{\tau}} / \partial \sigma_{A}\right)=0$. Here, $\psi[z]$ is the Digamma function [31]. Although the closed-form solution cannot be carried out, a simple relationship between the optimum HPBW and the misalignment $\phi_{0}$ can be found for a small misalignment in an approximate way.

Since the first-order derivative of the Digamma function $\psi[q](q \geq 0)$ is a decreasing function and goes to 0 in the infinity, the limit

$$
\begin{aligned}
\lim _{q \rightarrow+\infty}\left(\psi\left[\frac{1}{2}+q\right]\right. & -\psi[1+q]) \\
& =\lim _{q \rightarrow+\infty}\left(\psi[q]-\psi\left[\frac{1}{2}+q\right]\right)=0
\end{aligned}
$$

is valid. It can be seen that if the misalignment $\left|\phi_{0}\right| \rightarrow 0$, the parameter $q$ must be $q \rightarrow+\infty$ for the equality in (62) to be valid. Next, according to the property of the Digamma function $\psi[z+1]=\psi[z]+(1 / z)$, we have

$$
\left(\psi\left[\frac{1}{2}+q\right]-\psi[1+q]\right)=-\left(\psi[q]-\psi\left[\frac{1}{2}+q\right]\right)-\frac{1}{q} .
$$

Using (63), the relationship in (64) can be approximated by

$$
\psi\left[\frac{1}{2}+q\right]-\psi[1+q] \approx-\frac{1}{2 q}
$$

for a sufficiently large value of $q$. Combining the relationships (49), (62), and (65), the following approximation is achieved:

$$
\cos \frac{\sigma_{A[\mathrm{opt}]}}{2} \approx\left(\cos \phi_{0}\right)^{2 \ln 2} .
$$

Last, using $\cos ^{n} \phi_{0} \sim\left(1-\left(n \phi_{0}^{2} / 2\right)\right)$ for $\left|\phi_{0}\right| \rightarrow 0$, the optimum HPBW is related to a small misalignment $\phi_{0}$ by the following approximation:

$$
\sigma_{A[\mathrm{opt}]} \approx 2 \sqrt{2 \ln 2} \phi_{0} \approx 2.35 \phi_{0} .
$$

Fig. 6 depicts the theoretical relationship (62) and its approximate (67) between the optimum HPBW and the misalignment $\phi_{0}$, respectively, which indicates that (67) is a fairly good approximation in a large misalignment range.

\section{B. Uniform PAS in a Sphere}

If the scattered waves are uniformly distributed in a sphere, the optimum HPBW concerning the largest $K$-factor and RDS reduction can be achieved by solving

$$
\ln \cos \frac{\sigma_{A[\mathrm{opt}]}}{2}=\frac{\ln \cos ^{\ln 2} \phi_{0}}{1+\ln \cos \phi_{0}}
$$

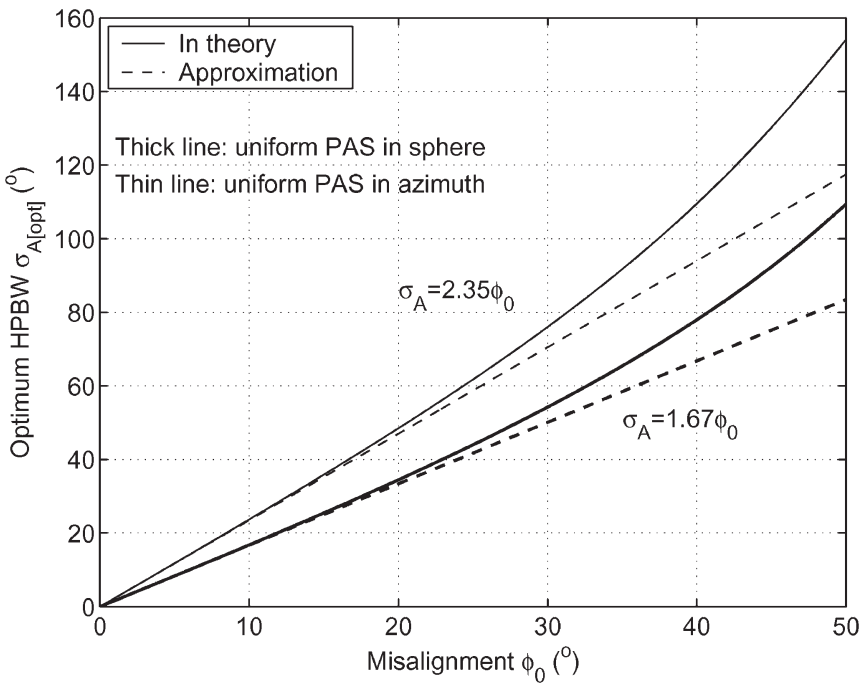

Fig. 6. Optimum HPBW $\sigma_{A[\mathrm{opt}]}$ versus the misalignment $\phi_{0}$ for the cases of uniform angular spectrum in azimuth and in 3-D for the scattered waves.

which is obtained by computing $\left(\partial G_{K} / \partial \sigma_{A}\right)=0$, $\left(\partial G_{\rho} / \partial \sigma_{A}\right)=0$, and $\left(\partial R_{\sigma_{\tau}} / \partial \sigma_{A}\right)=0$. Using $\ln \cos \phi_{0} \sim$ $-\left(\phi_{0}^{2} / 2\right)$ for $\left|\phi_{0}\right| \rightarrow 0$, we have

$$
\sigma_{A[\mathrm{opt}]} \sim \frac{2 \sqrt{2 \ln 2} \phi_{0}}{\sqrt{2-\phi_{0}^{2}}} \approx 2 \sqrt{\ln 2} \phi_{0} \approx 1.67 \phi_{0} .
$$

Fig. 6 depicts the theoretical relationship [see (68)] and its approximate [see (69)] between the HPBW and the misalignment $\phi_{0}$, respectively, which indicates that (69) is a fairly good approximation in a large misalignment range.

\section{APPENDIX B}

\section{AZIMUTH Scan RANGE AND ELEMENT BEAMWIDTH}

Here, we only investigate the relationship between the $3-\mathrm{dB}$ beam scan range in the azimuth plane and the antenna element beamwidth at the receiver side. The following results are also true for the scan range at the transmitter side. If the departure direction of the LOS wave is fixed at $\Omega_{t, 0}$, then from (53) and (54), the maximum gains of the $K$-factor and SNR, respectively, can be achieved as

$$
\begin{aligned}
\max \left\{G_{K}\right\} & =\frac{P^{2} Q^{2} A\left(\Omega_{t, 0}\right) A\left(\frac{\pi}{2}, 0\right)}{F_{t, C} F_{r, C}} \\
\max \left\{G_{\rho}\right\} & =\frac{\left(K \cdot \max \left\{G_{K}\right\}+1\right) F_{t, C} F_{r, C}}{P Q(K+1)}
\end{aligned}
$$

when the LOS wave arrives in the receiver at $\Omega_{r, 0}=((\pi / 2), 0)$. Now, the 3-dB scan range in the azimuth plane can be derived as

$$
\phi_{\text {scan }}=\left|\phi_{r, 0}^{U}-\phi_{r, 0}^{L}\right|
$$

where $\phi_{r, 0}^{U}$ and $\phi_{r, 0}^{L}$ are the solutions to $G_{\rho}=(1 / 2) \max \left\{G_{\rho}\right\}$ that is simplified as

$$
\frac{A\left(\frac{\pi}{2}, \phi_{r, 0}\right)}{A\left(\frac{\pi}{2}, 0\right)}=\frac{1}{2}-X
$$


where $X=1 /\left(2 K \max \left\{G_{K}\right\}\right)$. It can be seen in (73) that the azimuth scan range is never larger than the element HPBW because of $\left(A\left((\pi / 2), \phi_{r, 0}\right) / A((\pi / 2), 0)\right)=(1 / 2)$ at $\phi_{r, 0}=$ $\pm\left(\sigma_{A} / 2\right)$. Fig. 4(a) shows that for a fairly large number of antenna elements, a large $K$-factor gain can be achieved, which leads to $X \ll(1 / 2)$ for a fairly large $K$-factor. In this case, the azimuth scan range is approximated by the element beamwidth, i.e.,

$$
\phi_{\text {scan }} \approx \sigma_{A} \text {. }
$$

\section{ACKNOWLEDGMENT}

The authors would like to thank all the anonymous reviewers for their valuable comments.

\section{REFERENCES}

[1] C. Jakes, Microwave Mobile Communications. Hoboken, NJ: Wiley, 1974.

[2] J. D. Parsons, The Mobile Radio Propagation Channel. London, U.K.: Pentech, 1994.

[3] P. A. Bello, "Characterization of randomly time-variant linear channels," IEEE Trans. Commun., vol. COM-11, no. 4, pp. 360-393, Dec. 1963.

[4] H. Hashemi, "The indoor radio propagation channel," Proc. IEEE, vol. 81, no. 7, pp. 943-968, Jul. 1993.

[5] P. Höeher, "A statistical discrete-time model for the WSSUS multipath channel," IEEE Trans. Veh. Technol., vol. 41, no. 4, pp. 461-468, Nov. 1992

[6] E. Sousa, V. Jovanovic, and C. Daigneault, "Delay spread measurements for the digital cellular channel in Toronto," IEEE Trans. Veh. Technol., vol. 43, no. 4, pp. 837-847, Nov. 1994.

[7] R. Ertel, P. Cardieri, K. Sowerby, T. Rappaport, and J. Reed, "Overview of spatial channel models for antenna array communication systems," IEEE Pers. Commun., vol. 5, no. 1, pp. 10-22, Feb. 1998.

[8] T. Taga and T. Tanaka, "Delay spread reduction effect of beam antenna and adaptively controlled beam facing access system in urban line-of-sight street microcells," IEEE Trans. Veh. Technol., vol. 52, no. 4, pp. 761-771, Jul. 2003.

[9] M. Williamson, G. Athanasiadou, and A. Nix, "Investigating the effects of antenna directivity on wireless indoor communication at $60 \mathrm{GHz}$," in Proc. 8th IEEE Symp. PIMRC, Sep. 1997, vol. 2, pp. 635-639.

[10] P. Smulders, "Exploiting the $60 \mathrm{GHz}$ band for local wireless multimedia access: Prospects and future directions," IEEE Commun. Mag., vol. 40, no. 1, pp. 140-147, Jan. 2002.

[11] T. Manabe, Y. Miura, and T. Ihara, "Effects of antenna directivity and polarization on indoor multipath propagation characteristics at 60 GHz," IEEE J. Sel. Areas Commun., vol. 14, no. 3, pp. 441-448, Apr. 1996.

[12] Y. Sun, P. Hafezi, A. Nix, and M. Beach, "Indoor channel characterisation measurements with directional antennas for future high frequency ATM wireless access systems," in Proc. PIMRC, Helsinki, Finland, 1997, pp. $184-188$.

[13] J. Dabin, N. Ni, A. Haimovich, E. Niver, and H. Grebel, "The effects of antenna directivity on path loss and multipath propagation in UWB indoor wireless channels," in Proc. IEEE Conf. Ultra Wideband Syst. Technol., Nov. 2003, pp. 305-309.

[14] H. Yang, M. H. A. J. Herben, and P. F. M. Smulders, "Frequency selectivity of 60-GHz LOS and NLOS indoor radio channels," in Proc. IEEE VTC_-Spring, Melbourne, Australia, 2006, pp. 2727-2731.

[15] T. Taga, "Analysis for mean effective gain of mobile antennas in land mobile radio environments," IEEE Trans. Veh. Technol., vol. 39, no. 2, pp. 117-131, May 1990.

[16] A. Glazunov, "Theoretical analysis of mean effective gain of mobile terminal antennas in Ricean channels," in Proc. IEEE VTC_Fall, 2002, pp. 1796-1800.

[17] A. Glazunov, "Mean effective gain of user equipment antennas in double directional channels," in Proc. IEEE PIMRC, 2004, vol. 1, pp. $432-436$.

[18] E. Bonek and M. Steinbauer, "Double-directional channel measurements," in Proc. IEE 11th Int. Conf. Antennas Propag., Apr. 2001, vol. 1, pp. 226-230.
[19] M. Steinbauer, A. Molisch, and E. Bonek, "The double-directional radio channel," IEEE Antennas Propag. Mag., vol. 43, no. 4, pp. 51-63, Aug. 2001.

[20] B. H. Fleury, "First- and second-order characterization of direction dispersion and space selectivity in the radio channel," IEEE Trans. Inf. Theory, vol. 46, no. 6, pp. 2027-2044, Sep. 2000.

[21] R. Heddergott and P. Truffer, "Results of indoor wideband delay-azimuth-elevation measurements for stochastic radio channel modeling," AT\&T Bell Labs., Tech. Rep. COST 259 TD (99) 083, Sep. 1999. COST 259.

[22] K. Pedersen, P. E. Mogensen, and B. H. Fleury, "A stochastic model of the temporal and azimuthal dispersion seen at the base station in outdoor propagation environments," IEEE Trans. Veh. Technol., vol. 49, no. 2, pp. 437-447, Mar. 2000.

[23] Q. H. Spencer, B. D. Jeffs, M. A. Jensen, and A. L. Swindlehurst, "Modeling the statistical time and angle of arrival characteristics of an indoor multipath channel," IEEE J. Sel. Areas Commun., vol. 18, no. 3, pp. 347-360, Mar. 2000

[24] A. Kuchar, J.-P. Rossi, and E. Bonek, "Directional macro-cell channel characterization from urban measurements," IEEE Trans. Antennas Propag., vol. 48, no. 2, pp. 137-146, Feb. 2000.

[25] J. Laurila, K. Kalliola, M. Toeltsch, K. Hugl, P. Vainikainen, and E. Bonek, "Wideband 3-D characterization of mobile radio channels in urban environment," IEEE Trans. Antennas Propag., vol. 50, no. 2, pp. 233-243, Feb. 2002.

[26] S. Silver, Microwave Antenna Theory and Design. London, U.K.: Peregrinus, 1984.

[27] C. A. Balanis, Antenna Theory: Analysis and Design, 2nd ed. Hoboken, NJ: Wiley, 1997.

[28] H. J. Visser, Array and Phased Array Antenna Basics. Chichester, U.K.: Wiley, 2005

[29] K. Kalliola, K. Sulonen, H. Laitinen, O. Kivekas, J. Krogerus, and P. Vainikainen, "Angular power distribution and mean effective gain of mobile antenna in different propagation environments," IEEE Trans. Veh. Technol., vol. 51, no. 5, pp. 823-838, Sep. 2002.

[30] N. Blaunstein et al., "Signal power distribution in the azimuth, elevation and time delay domains in urban environments for various elevations of base station antenna," IEEE Trans. Antennas Propag., vol. 54, no. 10, pp. 2902-2916, Oct. 2006.

[31] M. Abramowitz and I. A. Stegun, Handbook of Mathematical Functions With Formulas, Graphs, and Mathematical Tables. New York: Wiley, 1984.

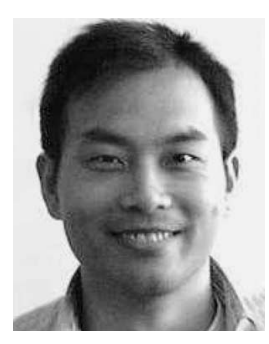

Haibing Yang (S'01-M'03) received the B.S. degree in detection and instrumentation and the M.Sc. degree in electrical engineering from Xidian University, Xi'an, China, in 1997 and 2000, respectively, and the degree of Professional Doctorate in Engineering (PDEng) from Eindhoven University of Technology (TU/e), Eindhoven, The Netherlands, in 2002.

From 1998 to 2000, he was with the National Key Laboratory of Radar Signal Processing, Xi' an, where he was involved in a project concerning the applications of neural networks in wireless communications. From 2001 to 2002, he was a Research Assistant with Philips Research Laboratory, Eindhoven, where he focused on Doppler cancellation for mobile DVB-T reception. Since 2003, he has been with the Radiocommunications Group, TU/e, where he is involved in the project Broadband Radio@Hand. He is currently involved in the Freeband project WiComm on $60-\mathrm{GHz}$ millimeter-wave communications. His research interests are in the areas of radio channel modeling and signal processing for wideband wireless communications.

Dr. Yang is a member of the Netherlands Electronics and Radio Society (NERG). 


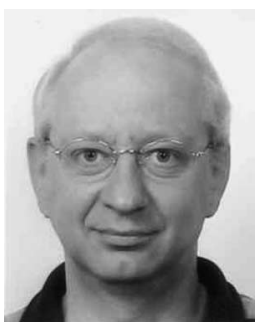

Matti H. A. J. Herben (S'80-M'83-SM'88) was born in Klundert, The Netherlands, in 1953. He received the M.Sc. degree (cum laude) in electrical engineering and the Ph.D. degree in technical sciences from Eindhoven University of Technology (TU/e), Eindhoven, The Netherlands, in 1978 and 1984 , respectively.

Since 1978, he has been with the Radiocommunications Group, TU/e, where he is currently an Associate Professor. His research interests and publications are in the areas of antennas, radio wave propagation, channel modeling for wireless communications, and atmospheric remote sensing.

Dr. Herben is a Treasurer of the IEEE Benelux Joint Chapter on Communications and Vehicular Technology, Associate Editor of the IEEE TRANSACTIONS on Antennas and Propagation, and a member of the Royal Institute of Engineers (KIvI), the Netherlands Electronics and Radio Society (NERG), and the Dutch National Committee of the International Radio Science Union (URSI).

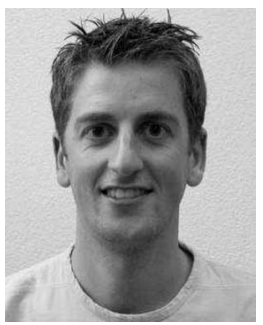

Iwan J. A. G. Akkermans (S'01) received the M.Sc. degree in electrical engineering in 2004 from Eindhoven University of Technology, Eindhoven, The Netherlands, where he is currently working toward the Ph.D. degree.

His research focuses on the design of antennas for broadband communication at millimeter-wave frequencies. His research interests include wireless power transfer, electromagnetic modeling, and antenna measurement.

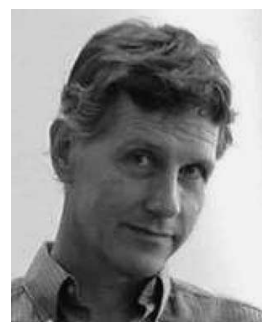

Peter F. M. Smulders (S'84-M'88-SM'97) received the M.Sc. and Ph.D. degrees in electrical engineering from Eindhoven University of Technology (TU/e), Eindhoven, The Netherlands, in 1985 and 1995, respectively.

In 1985, he was with the Propagation and Electromagnetic Compatibility Department, Research Neher Laboratories, Netherlands PTT, where he was doing research in the field of compromising emanation from civil data processing equipment. Since 1988, he has been with the Telecommunication Division, Eindhoven University of Technology. In addition to his lecturing duties, he performed $\mathrm{Ph} . \mathrm{D}$. research in the field of $60-\mathrm{GHz}$ broadband wireles LANs. His current work addresses the feasibility of low-cost low-power smallsized wireless LAN technology operating in the $60-\mathrm{GHz}$ frequency band. With this low-cost technology, it should be possible to serve an unprecedented maximum user data rate on the order of gigabits per second. His research interest that covers $60-\mathrm{GHz}$ physical and higher layers is reflected in numerous IEEE publications. He was involved in various research projects addressing $60-\mathrm{GHz}$ antennas and interworking (ACTS, MEDIAN) and 60-GHz propagation (MinEZ, Broadband Radio@Hand). He is also currently addressing baseband design in the context of $60-\mathrm{GHz}$ radio (Freeband, WiComm). As Project Manager of the SiGi-Spot project, his future research activities will range from $60-\mathrm{GHz}$ physical layer design to associated network aspects. 